\title{
CORRECTIONS TO:
}

\section{METAPLECTIC FORMS}

\author{
by D. A. KAZHDAN and S. J. PATTERSON
}

(Publications Mathématiques, 59 (I984), 35-142)

Unfortunately the argument on pp. I 1 9-120 of this paper were carried out too hastily as was pointed out to us by $T$. Suzuki. The assertion $\left\langle\lambda_{\eta_{v}}, v_{0, v}\right\rangle=0 \quad\left(\eta \notin \widetilde{\mathbf{H}}_{*, v}\right)$ is false which necessitates the following additional argument. For $v \notin \mathrm{S}$ for which one has $|n|_{v}=\mathrm{I}$ and $\omega_{*, v} \mid \widetilde{\mathbf{H}}_{*, v} \cap \mathrm{K}_{v}^{*}=\mathrm{I}$ we have to have for consistency

$$
\sum_{\eta_{v} \in \tilde{\mathrm{H}}_{*, v} \backslash \tilde{\mathrm{H}}_{v}} \mathbf{c}_{\mathrm{B} \cup\{v\}}\left(\eta \times \eta_{v}\right)\left\langle\lambda_{\eta_{v}}, v_{0, v}\right\rangle=\mathbf{c}_{\boldsymbol{S}}(\eta) \text {. }
$$

By Theorem I.4.2 and Proposition I.2.4 the left-hand side is equal to

$$
\mathrm{c}_{\mathrm{S} \cup\{v\}}(\eta \times \mathrm{I})\left(\mathrm{I}-q_{v}^{-1}\right)\left(\mathrm{I}-q_{v}^{-2}\right) \ldots\left(\mathrm{I}-q_{v}^{-r}\right) /\left(\mathrm{I}-q_{v}^{-1}\right)^{r} .
$$

Thus we can define

where

$$
\mathbf{c}(\eta)=\lim _{\mathbf{S} \uparrow} \mathbf{c}_{\mathrm{S}}(\eta) \mathrm{T}(\mathrm{S})
$$

$$
\mathrm{T}(\mathrm{S})=\prod_{\substack{v \in \mathrm{S} \\ v+\infty}}\left(\mathrm{I}+q_{v}^{-1}\right)\left(\mathrm{I}+q_{v}^{-1}+q_{v}^{-2}\right) \ldots\left(\mathrm{I}+q_{v}^{-1}+\ldots+q_{v}^{-(r-1)}\right) .
$$

The limit stabilises for large enough $\mathrm{S}$. With this definition the formula of Theorem II. 2.2 should read

$$
\int_{\mathrm{N}_{*}^{*}, k \backslash \mathrm{N}_{*}^{*}, \mathrm{~A}} \bar{e}(n) \theta\left(n, f_{0}\right) d n=\lim _{\mathrm{S} \uparrow} \sum_{n \in \tilde{\mathrm{H}}_{*, \mathrm{~A}}(\mathrm{~S}) \backslash \tilde{\mathrm{H}}_{\mathrm{A}}(\mathrm{S})} \mathbf{c}(\eta) \mathrm{T}(\mathrm{S})^{-1} \prod_{\nu \in \mathrm{S}}\left\langle\lambda_{\eta_{v}}, f_{*, v}\right\rangle .
$$

The same modifications should be made to Theorem II.2.3 and the discussion on p. 13o. The second author would like to point out that the same applies to the survey "Whittaker Models of Generalised Theta Series » in Sém. Théorie des Nombres de Paris 1982-1983, Birkhäuser, I984, pp. 199-232. This applies especially to $\S 4 \cdot 7$; the correction is already included in $\S 5.6$.

The second author is compelled to admit that in this survey he was carried away by a now inexplicable bout of optimism; the conjecture proposed in $\S 6$ cannot be true as stated. Nevertheless it does appear to be true when the functions on either side are restricted to an appropriately small subset of $\widetilde{H}_{A}$ as $T$. Suzuki has kindly informed us. 